\title{
AKTiveSA: Supporting Civil-Military Information Integration in Military Operations Other than War
}

\author{
Paul R. Smart, Alistair Russell and Nigel R. Shadbolt \\ School of Electronics and Computing Science, University of Southampton, Southampton, SO17 1BJ, UK \\ $\{$ ps02v, ar5, nrs\}@ecs.soton.ac.uk
}

\begin{abstract}
The ability to integrate information from a variety of sources is a key ingredient of enhanced situation awareness in both conflict and non-conflict situations. Information integration is particularly important in Military Operations Other Than War (MOOTW) contexts because the range of relevant information sources is considerably more diverse than that seen in more conventional warfighting operations. In this paper we provide an overview of a technical demonstrator system (the AKTiveSA TDS), which was developed as part of the UK MoD's Defence Technology Centre (DTC) initiative. The demonstrator combines a variety of semantic technologies with advanced modes of visualization and interaction in order to highlight how extant Semantic Web technologies can be used to improve situation awareness and facilitate information integration in a simulated humanitarian relief scenario.
\end{abstract}

\section{INTRODUCTION}

Military Operations Other Than War (MOOTW) present a number of challenges to military agencies in terms of both information exploitation and inter-agency collaboration. MOOTW operations often require the integration of information from a variety of physically disparate and semantically heterogeneous information resources and, in many MOOTW operational contexts, such as humanitarian relief, military agencies are often required to work alongside other, non-military agencies, which raises issues concerning the effectiveness of inter-agency communication and information exchange mechanisms. In the context of the UK Data and Information Fusion (DIF) Defence Technology Centre (DTC) initiative we have been engaged in a program of research, called AKTiveSA, which aims to investigate issues relating to the effective inter-operation of both military and non-military agencies in a variety of MOOTW contexts. As part of our work we have developed a simulated humanitarian relief scenario to support subsequent knowledge engineering activities and also to provide a basis for technical demonstration. The scenario features a number of humanitarian incidents based around an earthquake in the Ghazni region of Afghanistan, and these events are superimposed on a backdrop of ongoing military conflict involving air strikes and ground manoeuvres by coalition forces against elements of the former Taliban regime. Formal ontologies, developed using the Web Ontology Language (OWL), were used to support the semantic annotation of various scenario-relevant information resources and to provide a representational substrate for inference-mediated information fusion processes. In addition to providing an effective solution for search and retrieval operations using semantic query languages such as SPARQL ${ }^{1}$, formal ontological representations were also used to support a number of visualization capabilities including the generation of MIL 2525B military symbols [1] and map overlays that juxtapose both military and non-military information. In this paper, we describe the ontologies that were developed in the context of the project and demonstrate how these can be used to support integrated information displays in the context of a technical demonstrator system: the AKTiveSA TDS. The system described herein exploits a variety of semantic technology components to highlight strategies for semantically-mediated information integration and display, especially in situations where information about the disposition of coalition military assets needs to be aligned with situation-relevant civil-intelligence. Although, our development work with respect to the AKTiveSA TDS is ongoing, this paper provides an overview of current system capabilities and describes how these capabilities will be extended in future development efforts. The overview of system capabilities presented here extends an earlier characterization of a prototype system, which was described in Smart et al [2].

\section{ONTOLOGY DEVELOPMENT}

The basis of our approach in developing semanticallymediated approaches to information integration consists in the provision of formal ontologically-motivated characterizations of the problem domain. Due to the desired support for a variety of knowledge-oriented processes, we required the use of a suitably rich and expressive medium for knowledge representation. Recently, attempts to provide a set of representational formalisms for the communication of ontological structures within the framework of the Semantic Web have coalesced around the Web Ontology Language (OWL) $[3,4]$. We elected to use this language as the representational medium for our ontology engineering activities, in part due to its endorsement by the World Wide Web Consortium (W3C), its close alignment with Resource Description Framework (RDF) and its level of semantic expressivity (which supports a variety of types of automated reasoning, e.g. subsumption reasoning).

A number of ontologies were developed as part of the knowledge engineering initiative for the AKTiveSA project. These included, but were not limited to:

\footnotetext{
${ }^{1}$ http://www.w3.org/TR/rdf-sparql-query/
} 
1. Geography: This ontology deals with all the geographical aspects of the problem domain. It encompasses a wide variety of conceptualisations including terrain features, transport routes, rivers, shorelines, terrain elevation data, etc.

2. Transportation: This ontology covers all aspects of transportation in the problem domain. This overlaps, to some extent, with the geography ontology in the sense that transportation routes, e.g. airways and roads, may also be considered elements of the geographical (geo-spatial) domain.

3. Meteorology: This ontology deals with all aspects of the climate and weather. The meteorology ontology is important in enabling the system to interpret and utilize information derived from local weather reports and forecasts as well as long term data about regional rainfall, snowfall, seasonal temperature, etc.

4. Humanitarian Aid: This ontology covers information of relevance to humanitarian operations, i.e. humanitarian hazards (e.g. floods), humanitarian organizations, humanitarian aid programs, humanitarian aid workers, etc.

5. Military Entities: This ontology includes all relevant conceptualisations in the military domain, including tactical operational areas and zones, military platforms, intelligence information, weapons, etc.

The results of the knowledge engineering initiative were presented in the form of a knowledge web (see Figure 1), which provided a web-based medium for browsing both domain ontologies and knowledge sources used as part of the ontology engineering initiative. The knowledge web was used by military Subject Matter Experts (SMEs) as part of the knowledge validation process.

\section{TEChNiCAL Demonstrator SySTEM}

To showcase the role played by semantically-enriched representations in supporting information integration and situation awareness, we developed a technical demonstrator system using a variety of technology components. These components are described in subsequent sections.

\subsection{Knowledge Repository}

The AKTiveSA Knowledge Repository is a key element of the AKTiveSA TDS and corresponds to an instance of the AKT 3Store [5]. The 3Store combines an RDF tripleStore with a SPARQL query engine that promotes the efficient storage and retrieval of RDF metadata. The 3Store is implemented on top of a MySQL database engine, which can be manipulated using conventional queries formulated in SQL. However, in order to provide more sophisticated query capabilities, the 3Store also incorporates a SPARQL interface. The 3Store SPARQL engine transforms a

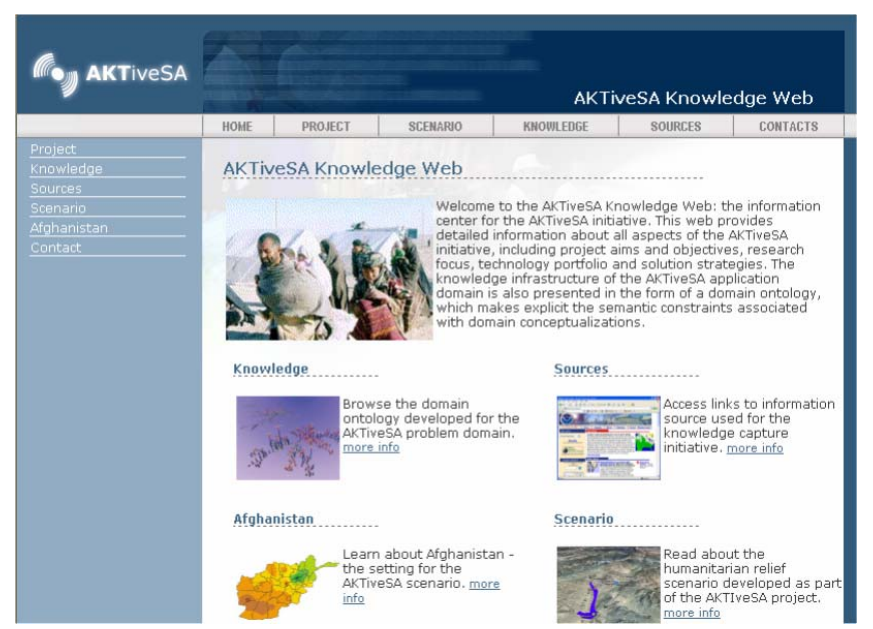

Figure 1: AKTiveSA Knowledge Web

SPARQL query into a SQL query, which can then be executed against the relational database representation of the RDF data to return a query result. The AKTiveSA Knowledge Repository is used as the storage medium for the entire knowledge infrastructure of the AKTiveSA application domain. It contains all the ontologies and metadata associated with the application, including both schematic knowledge (classes) and knowledge objects (individuals).

\subsection{Military Symbology Browser}

To provide a common representation of the operational environment, and to support a common understanding of the situation picture, we aimed to exploit standard military symbols for the graphical representation of military entities. The MIL 2525B symbol specification [1] was used as the basis for creating military symbols using ontological characterizations of specific military entities. Essentially, all military entities defined within the scenario were associated with ontology elements in the Knowledge Repository, and this information served as the basis for the dynamic creation of graphic symbols via a web service interface. The web service in this case accepted, as input, a Uniform Resource Identifier (URI), which uniquely identified the target entity in the context of the AKTiveSA ontology infrastructure. The

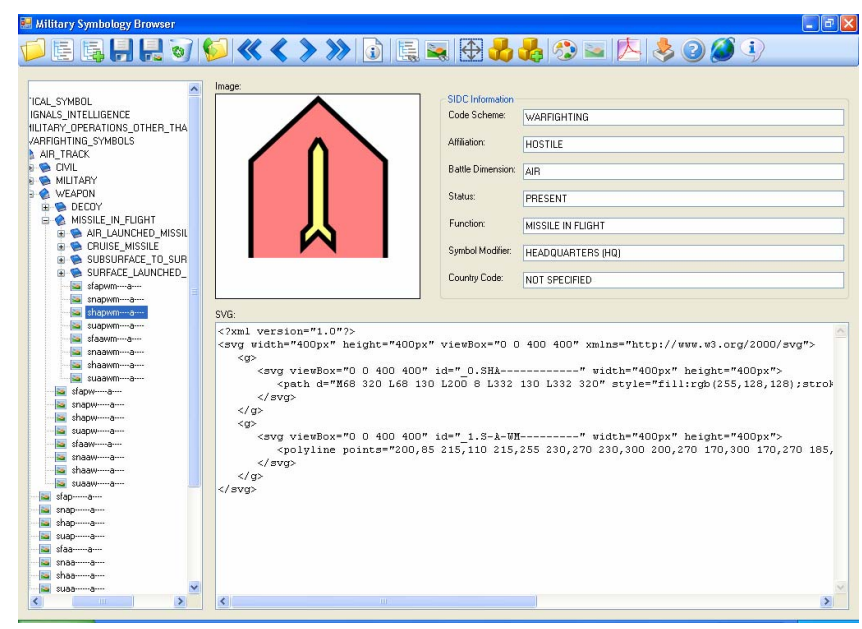

Figure 2: Military Symbology Browser 
service then retrieved relevant information about the entity from the Knowledge Repository and used this information to determine the Symbology Identification Code (SIDC) for the entity ${ }^{2}$. This information was in turn used to programmatically create an appropriate graphic symbol using the Military Symbology API, a code component specifically engineered for military symbol generation in the context of the AKTiveSA project. Once created, the web service returned an image to the AKTiveSA Client Application (see Section 3.5) for display in the user interface. The technology portfolio of the AKTiveSA initiative includes the Military Symbology Browser (see Figure 2), a stand-alone application that exposes the functionality of the aforementioned Military Symbology API. This application demonstrates the automatic generation of military symbol hierarchies, the use of Scalable Vector Graphics (SVG) to represent graphic elements, the alignment of graphic symbols with SIDC information, and the automatic rendering of SVG elements to image files using GDI+. The application and the associated web service wrapper to the Military Symbology API could be used, in a highly reusable fashion, to support ontology-driven military symbol generation in a variety of application contexts.

\subsection{AKTive8 Semantic Web API}

Many of the knowledge and reasoning capabilities of the AKTiveSA TDS rely on the AKTive8 Semantic Web API. This is a .NET class library that provides a generic and reusable framework for processing Semantic Web data. It was developed in the context of the AKTiveSA initiative and encapsulates the functionality for knowledge representation, publishing and reasoning in the context of the Semantic Web. Within the context of the AKTiveSA TDS, the AKTive8 API is used to provide access to the AKTiveSA Knowledge Repository, to execute semantic queries, and to provide an interface between the AKTiveSA Client Application and other technology components, e.g. the AKTiveSA Scenario Generator.

\subsection{Scenario Generator}

The AKTiveSA TDS was developed to showcase how semantic technologies could be used to improve information integration in respect of civil-military operational contexts. As part of the development effort towards this goal it was necessary to define a scenario that could be used for visualization, demonstration and evaluation purposes. The chosen scenario features a number of humanitarian incidents, including the collapse of the Band Sultan dam in Ghazni province. These humanitarian incidents are superimposed on an ongoing backdrop of military conflict (based on coalition military operations against Taliban insurgents) in the South-Eastern region of Afghanistan. Although the scenario was based on real-world events, most of the information resources for our scenario were largely contrived. In particular, we manually created the web pages,

\footnotetext{
2 The SIDC is a unique alphanumeric identifier, which serves to uniquely identify each symbol within the MIL 2525B symbology specification.
}

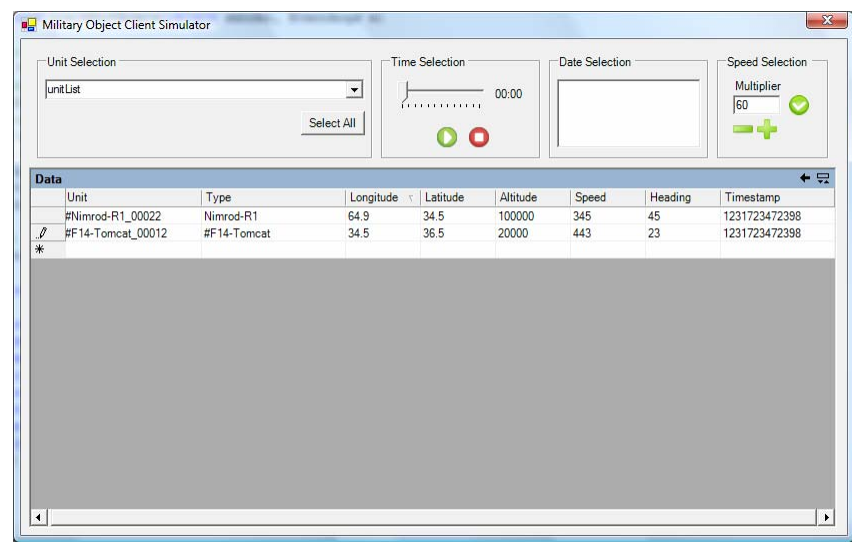

Figure 3: Scenario Generator Tool

text reports, RSS feeds, etc., which were necessary to support the scenario timeline. In most cases, however, these resources were derived from actual information resources publicly available via the web.

A scenario generator tool was developed to coordinate the generation of information feeds and event triggers in respect of the scenario. Figure 3 shows a screenshot of the scenario generator tool. The tool reads information from a flat relational database, which contains information about the disposition of military and non-military units. It then feeds this information into an AKTive8 API web service for use within the AKTiveSA Client Application. The scenario generator tool can be used to control the speed of the scenario timeline as well as the current position within the timeline - changes to the current time cause the Scenario Generator to update information about all scenario objects (military units, refugee convoys, etc.) visible via the user interface.

\subsection{AKTiveSA Client Application}

The AKTiveSA Client Application (see Figure 4) is the centrepiece of the AKTiveSA TDS. It hosts the NASA Worldwind $^{3}$ visualization component, which co-opts both photorealistic satellite imagery with digital terrain elevation data to provide, what is in effect, a three dimensional model of the Earth's surface. The AKTiveSA Client Application extends the Worldwind component (called the WorldView, in the context of our AKTiveSA component hierarchy) by providing a number of advanced interface components, including overlays that can host multiple of types of filtered information content (e.g. military symbols, maps, images, etc.).

\section{Architecture}

The AKTiveSA TDS architecture is based on a client-server model, wherein the AKTive8 API mediates access to a shared AKTiveSA Knowledge Repository that is used by (perhaps) multiple instances of the AKTiveSA Client Application. This architecture allows for the provision of shared views of the current operational picture by both military and civilian agencies (i.e. views can be shared

\footnotetext{
${ }^{3}$ http://worldwind.arc.nasa.gov/
} 

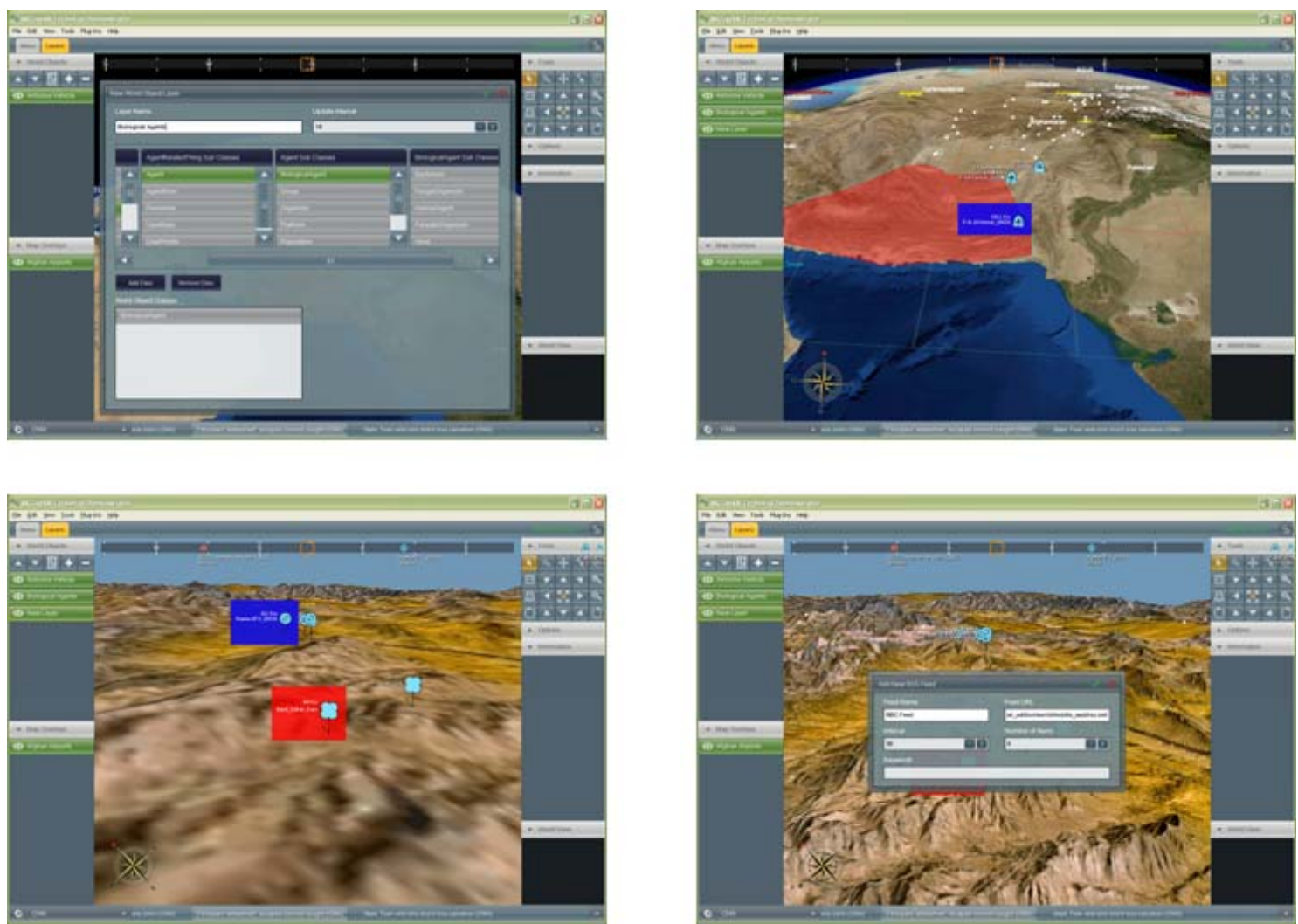

Figure 4: AKTiveSA Client Application

between multiple instances of the AKTiveSA Client Application running within a single networked environment).

Each application instance, in this case, provides editing capabilities, which allows authorized users to update the contents of the Knowledge Repository, thus changing the knowledge infrastructure of the situation picture.

\section{View Manipulation}

Each AKTiveSA Client Application provides multiple opportunities for manipulation of the WorldView. These actions enable the user to change the camera view angle, tilt-level, elevation level, etc. The current implementation assumes that user interaction is primarily mediated via standard input devices, such as keyboard and mouse; however, alternative modes of user interaction could be achieved, and in some situations these might be considered preferable. One notable example here concerns the use of large multi-touch screens which provide users with intuitive modes of interaction and, in some cases at least, also permit multiple users to work simultaneously with different parts of the application interface [6]. Touch-screen control of the user interface will often emulate that achieved using the mouse, so, for example, touching and dragging the WorldView will emulate a panning operation, while touching and dragging the fingers in a radial direction will emulate a zoom-in or zoom-out operation.

\section{RSS Ticker}

The AKTiveSA Client Application includes an RSS ticker control, which is designed to display information from various, user-selected news feeds. The summary for each news item is displayed in a scrolling marquee at the bottom of the user interface and each summary is associated with a 'More Information Button', which, when clicked, will open a dialog box to display the entire news article. Items that have already been displayed in this way are rendered using a white font, while items that have not previously been displayed are rendered using a red font.

\section{Image Overlays}

Image overlays represent visual information about areas of the terrain as rendered in the WorldView component. Image overlays can include both vector based graphics (for regionbased information, such as population density or language distribution maps), or additional satellite imagery layers. Such overlays provide a means to easily extend the types of information content that can be displayed using the WorldView component.

\section{Temporal Dynamics}

The AKTiveSA Client Application provides two means to manipulate the scenario timeline in order to gain a view of past and future (predicted) states. The movement toolbar displays a visible movement trail, which indicates the past and predicted movement of a selected entity (e.g. a military unit) within the WorldView display (see Figure 5). This 


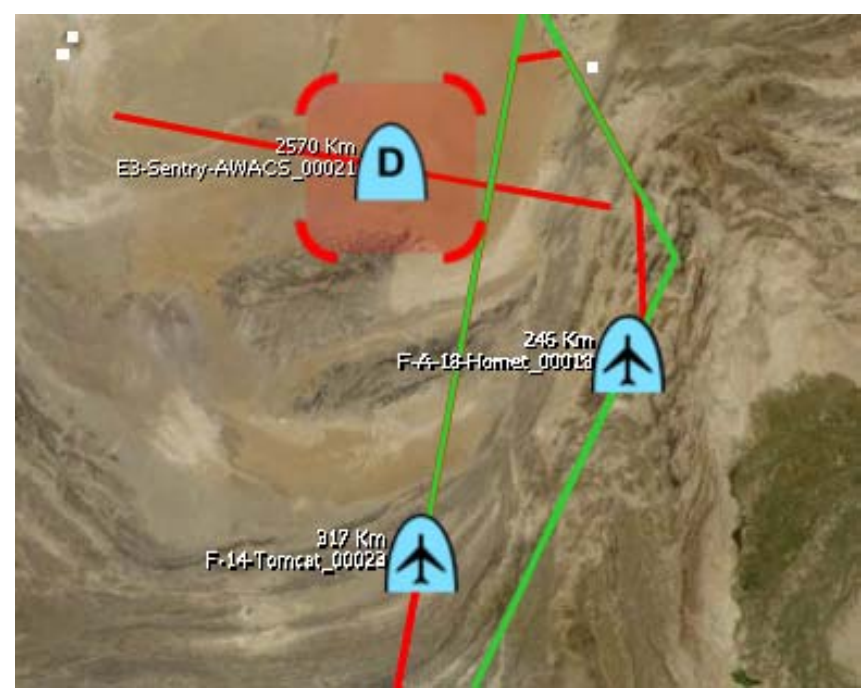

Figure 5: Entity Movements

visual aid facilitates an understanding of the temporal evolution of the current situation picture both in terms of past situations and (likely) future situations. A second tool, the time gesture tool, compliments the movement toolbar by allowing users to use simple input gestures to alter the current time setting. Horizontal movements from left to right across the screen, using either the mouse (or fingers on a touch-screen interface) advances the temporal position forward in time, whereas a gesture from right to left will cause the time setting to regress. Combining horizontal gestures with vertical gestures allows the speed of temporal progress to be manipulated: a movement from the bottom of the screen to the top will increase the speed at which the time changes, allowing for large jumps in temporal position; movements from the top of the screen to the bottom will decrease the speed at which the time changes, allowing for fine-tuning of the temporal setting.

\section{Knowledge Monitors}

Knowledge Monitors are technology components that serve as daemons responsible for the detection of knowledge-rich contingencies and the execution of knowledge processing actions. As their name suggests, Knowledge Monitors constantly monitor the evolving knowledge infrastructure of the AKTiveSA application domain. Each instantiated monitor is associated with a set of conditions and actions. When the conditions have been satisfied, the monitor invokes its associated actions, which typically serve to increase the situation awareness of the end user via alerts, notifications, status reports, emails or RSS feeds. Knowledge monitors can be created by end-users to detect and monitor interesting or important situation contingencies and they therefore serve as useful devices for event-driven processing and improved situation awareness.

\section{Conclusion}

The AKTiveSA initiative focuses on the development of technology to improve information integration and situation awareness in a variety of civil-military operational contexts.
To this end we have adapted a range of semantic technologies to demonstrate how semantically-enriched representational schemes can be used to assimilate information, facilitate search and retrieval and deliver operationally-useful decision outcomes in the context of a simulated humanitarian relief scenario. In addition to its ability to provide a sufficiently expressive medium for the representation of knowledge-rich contingencies, we also argue that the provision of semantically-enriched information can be used to filter information so as to avoid situations of information overload that might otherwise result from unrestricted access to large-scale, semantically heterogeneous information environments.

The AKTiveSA TDS provides a platform for demonstrating semantically-mediated modes of information integration and aggregation in the context a simulated humanitarian relief scenario. Operational contexts that require civil-military cooperation necessitate the exploitation (and exchange) of information that is considerably more diverse than that seen in conventional war-fighting contexts. For example, humanitarian relief operations, like that described in our scenario, necessitate the integration and juxtaposition of a variety of forms of information, including (but not necessarily limited to) information about refugee movements, the disposition of hostile forces, the status of ongoing offensive operations, the intentions and activities of humanitarian aid agencies, weather conditions, terrain features and the navigational status of key transport routes. The exploitation of such information for the purposes of operationally-effective modes of planning and decisionmaking is facilitated, we argue, by the use of semanticallyenriched representational schemes (that support the rapid search and retrieval of information) in conjunction with flexible modes of visualization and interaction (that present the user with an opportunity to overlay and juxtapose multiple types of information in the context of an integrated display environment).

The technology development effort associated with the AKTiveSA initiative is ongoing, and the current paper has provided only a snapshot of system capabilities at an intermediate point in the development timeline. Our future development efforts will focus on a number of enhancements to the current system, including the provision of a virtual adviser component (an animated virtual agent providing vocal prompts and alerts), a reasoning subsystem component (providing decision support with regard to selected knowledge-intensive tasks, e.g. humanitarian needs assessment), a resource annotation component (which will use natural language processing technologies to assist with the classification and semantic annotation of textual resources), and a graphical query designer component (to enable end-users to construct semantic queries in an intuitive and visually-oriented manner). These enhancements will, we hope, extend the applicability of the system to situations and domains beyond civil-military operational contexts. For example, we believe the current research could be usefully applied to any domain requiring 
information integration with respect to heterogeneous information sources for the purposes of enhanced situation awareness. These could include the emergency services, search and rescue operations [7], e-Health [8] and homeland security [9].

\section{ACKNOWLEDGMENT}

This work was funded by a grant awarded to General Dynamics UK Ltd and the University of Southampton as part of the Data and Information Fusion Defence Technology Centre initiative.

\section{REFERENCES}

[1] "Department of Defense Interface Standard: Common Warfighting Symbology," Department of Defense, USA MIL STD 2525B, 2005.

[2] P. R. Smart, N. R. Shadbolt, L. A. Carr, and m. c. schraefel, "Knowledge-Based Information Fusion for Improved Situational Awareness," presented at 8th International Conference on Information Fusion, Philadelphia, USA., 2005.

[3] G. Antoniou and F. van Harmelen, "Web Ontology Language: OWL," in Handbook on Ontologies, S. Steffen and R. Studer, Eds. Berlin: Springer Verlag, 2003.

[4] D. L. McGuiness and F. van Harmelen, "OWL Web Ontology Language Overview," World Wide Web Consortium, 2004. http://www.w3.org/TR/owl-features/

[5] S. Harris and N. Gibbins, "3store: Efficient Bulk RDF Storage," presented at 1st International Workshop on Practical and Scalable Semantic Web Systems, Sanibel Island, Florida, USA, 2003.

[6] J. Y. Han, "Low-cost multi-touch sensing through frustrated total internal reflection," presented at 18th Annual ACM Symposium on User Interface Software and Technology, Seattle, Washington, USA, 2005.

[7] "CoSAR-TS - Coalition Search and Rescue - Task Support Website." http://www.aiai.ed.ac.uk/project/cosar-ts/

[8] N. R. Shadbolt, P. Lewis, S. Dasmahaptra, D. Dupplaw, B. Hu, and H. Lewis, "MIAKT: combining grid and web services for collaborative medical decision making," presented at 2nd eScience All Hands Meeting, Nottingham, England, UK, 2004.

[9] "Semantic Web Applications for National Security - Conference Website." http://www.schafertmd.com/swans/ 